\title{
HAMILTONIAN CYCLES IN THE GENERATING GRAPHS OF FINITE GROUPS
}

\author{
T. BREUER, R. M. GURALNICK, A. LUCCHINI, A. MARÓTI, G. P. NAGY
}

\begin{abstract}
For a finite group $G$ let $\Gamma(G)$ denote the graph defined on the nonidentity elements of $G$ in such a way that two distinct vertices are connected by an edge if and only if they generate $G$. In this paper it is shown that the graph $\Gamma(G)$ contains a Hamiltonian cycle for many finite groups $G$.
\end{abstract}

\section{INTRODUCTION}

For a finite group $G$ let $\Gamma(G)$ denote the graph defined on the non-identity elements of $G$ in such a way that two distinct vertices are connected by an edge if and only if they generate $G$. The graph $\Gamma(G)$ is called the generating graph of $G$. The generating graph was investigated in [15], [16], and [17]. For example, in [16], it is shown that for a nilpotent by nilpotent finite group $G$ the clique number of $\Gamma(G)$ is equal to the chromatic number of $\Gamma(G)$.

In the literature many deep results about finite simple groups $G$ can equivalently be stated as theorems about $\Gamma(G)$. Three examples are given. Guralnick and Shalev [10] showed that for sufficiently large $G$ the graph $\Gamma(G)$ has diameter at most 2 . Guralnick and Kantor [9] showed that there is no isolated vertex in $\Gamma(G)$. Finally, Breuer, Guralnick, Kantor [4] showed that the diameter of $\Gamma(G)$ is at most 2 for all $G$.

In this paper those finite groups $G$ are considered for which $\Gamma(G)$ contains a Hamiltonian cycle. The following proposition reduces the investigations to those non-solvable groups $G$ for which $G / N$ is cyclic for any non-trivial normal subgroup $N$ of $G$.

Proposition 1.1. Let $G$ be a finite solvable group that has at least 4 elements. Then the graph $\Gamma(G)$ contains a Hamiltonian cycle if and only if $G / N$ is cyclic for all non-trivial normal subgroups $N$ of $G$.

The three main results of this paper are Theorems 1.2, 1.3, and 1.4.

Theorem 1.2. For every sufficiently large finite simple group $G$, the graph $\Gamma(G)$ contains a Hamiltonian cycle.

Theorem 1.3. For every sufficiently large symmetric group $S_{n}$, the graph $\Gamma\left(S_{n}\right)$ contains a Hamiltonian cycle.

Theorem 1.4. For every sufficiently large non-abelian finite simple group $S$, the graph $\Gamma\left(S \prec C_{m}\right)$ contains a Hamiltonian cycle, where $m$ denotes a prime power.

\footnotetext{
${ }^{1}$ The second author was partially supported by NSF grant DMS 0653873.

${ }^{2}$ The research of the fourth author was supported by a Marie Curie International Reintegration Grant within the 7th European Community Framework Programme and partially by grants OTKA T049841 and OTKA NK72523.

${ }^{3}$ Mathematics Subject Classification 2010: 20P05, 05C45

Date: 2nd of December, 2009.
} 
The proofs of Theorems 1.2, 1.3, and 1.4 depend heavily on Liebeck, Shalev [13], Fulman, Guralnick [6], Babai, Hayes [1], and Luczak, Pyber [18].

Theorem 1.5. Let $G$ be a sporadic simple group or the automorphism group of a sporadic simple group. Then the graph $\Gamma(G)$ contains a Hamiltonian cycle.

Based on Proposition 1.1, Theorems 1.2, 1.3, 1.4, 1.5, and some computer calculations performed by GAP [8] (see Section 8), the following conjecture is proposed.

Conjecture 1.6. Let $G$ be a finite group with at least 4 elements. Then the graph $\Gamma(G)$ contains a Hamiltonian cycle if and only if $G / N$ is cyclic for all non-trivial normal subgroups $N$ of $G$.

Conjecture 1.6 is related to Conjecture 1.8 (and the following paragraph) of [4]. Indeed, Burness, Guest, and Guralnick [5] are working on the problem of proving that $\Gamma(G)$ has no isolated vertex and indeed has diameter at most 2 if and only if $G / N$ is cyclic for every non-trivial normal subgroup $N$ of $G$. Moreover, the problem has been reduced to the case where $G$ is almost simple.

Problem 8.5 of The Kourovka Notebook [12] posed by M. R. Vaughan-Lee in 1982 is the following. Prove that if $G$ is a finite group, $F$ is any field, and $V$ is a non-trivial irreducible $F G$-module then

$$
\frac{1}{|G|} \sum_{g \in G} \operatorname{dim}(\operatorname{fix}(g)) \leq \frac{1}{2} \operatorname{dim}(V) .
$$

This was proved in case $(|G|,|V|)=1$ and also for solvable groups $G$ by Neumann and Vaughan-Lee in [19]. Later, Segal and Shalev [20] showed that, in general, the average dimension of fixed point spaces of elements of $G$ on $V$ is at most $(3 / 4) \operatorname{dim}(V)$. Finally, Isaacs, Keller, Meierfrankenfeld, Moretó [11] proved, in a slightly more general setting, that the average dimension of fixed point spaces of elements of $G$ on $V$ is at most $((p+1) /(2 p)) \operatorname{dim}(V)$ where $p$ denotes the smallest prime divisor of $|G|$. In this paper we show the following.

Proposition 1.7. Let $V$ be an irreducible $F G$-module of dimension at least 2 for some field $F$ and some finite group $G$. For an arbitrary element $g$ in $G$ let $d(g)$ denote the dimension of the largest eigenspace of $g$ on $V$. Suppose that the graph $\Gamma(G)$ contains a Hamiltonian cycle. Then

$$
\frac{1}{(|G|-1)} \sum_{1 \neq g \in G} d(g) \leq \frac{1}{2} \operatorname{dim}(V) .
$$

\section{GRAPHS}

A Hamiltonian cycle is a cycle in an undirected simple graph which visits each vertex exactly once. A graph is called Hamiltonian if it contains a Hamiltonian cycle. The problem of determining whether a graph is Hamiltonian is NP-complete and is a special case of the travelling salesman problem.

There are many ways to show that a given graph is Hamiltonian. First of all, sometimes it is possible just to exhibit a Hamiltonian cycle in the graph. This is the case for the graph $\Gamma(G)$ when $G$ is a solvable group of order at least 4 with the property that $G / N$ is cyclic for every non-trivial normal subgroup $N$ of $G$ (see Section 3).

A simple graph with $m$ vertices and list of vertex degrees $d_{1} \leq \ldots \leq d_{m}$ satisfies Pósa's criterion if $d_{k} \geq k+1$ for all positive integers $k$ with $k<m / 2$. By Exercise 10.21 (b) of [14], a graph contains a Hamiltonian cycle if it satisfies Pósa's criterion. 
It is shown in Sections 4 and 5 that $\Gamma(G)$ satisfy Pósa's criterion for almost all (if not all) finite simple groups $G$ of orders at least 5 .

For a simple graph $\Gamma$ with $m$ vertices let $d(\Gamma, v)$ denote the degree of the vertex $v$. The closure $\operatorname{cl}(\Gamma)$ of $\Gamma$ is the graph (on the same set of vertices) constructed from $\Gamma$ by adding for all non-adjacent pairs of vertices $u$ and $v$ with $d(\Gamma, u)+d(\Gamma, v) \geq m$ the new edge $u v$. One of the best characterization of Hamiltonian graphs is

Theorem 2.1 (Bondy, Chvátal, [2]). A graph is Hamiltonian if and only if its closure is Hamiltonian.

Theorem 2.1 is first applied in Section 6 of this paper.

For a simple graph $\Gamma$, let us set $\mathrm{cl}^{(1)}(\Gamma)=\operatorname{cl}(\Gamma)$ and inductively set $\mathrm{cl}^{(i)}(\Gamma)=$ $\mathrm{cl}\left(\mathrm{cl}^{(i-1)}(\Gamma)\right)$ for every positive integer $i$ larger than 1 .

A simple graph with $m$ vertices and list of vertex degrees $d_{1} \leq \ldots \leq d_{m}$ satisfies Chvátal's criterion if whenever $k$ is so that $d_{k} \leq k<m / 2$ it follows that $d_{m-k} \geq$ $m-k$. By Exercise 10.21 (d) of [14], a graph contains a Hamiltonian cycle if it satisfies Chvátal's criterion. In Section 6 it is shown that for every sufficiently large symmetric group $S_{n}$ the graph $\mathrm{cl}^{(3)}\left(\Gamma\left(S_{n}\right)\right)$ satisfies Chvátal's criterion.

\section{Solvable Groups}

In this section Proposition 1.1 is shown. Let $G$ be a finite solvable group with at least 4 elements.

If $\Gamma(G)$ contains a Hamiltonian cycle, then there is no isolated vertex in $\Gamma(G)$, hence $G / N$ must be cyclic for all non-trivial normal subgroups $N$ of $G$. It is sufficient to show the other implication. Suppose that $G$ is a finite group with the property that $G / N$ is cyclic for all non-trivial normal subgroups $N$ of $G$.

If $G$ is cyclic, then any generator $g$ of $G$ is connected to every other vertex of $\Gamma(G)$ and $g^{1}, g^{2}, \ldots, g^{n-1}, g^{1}$ determines a Hamiltonian cycle in $\Gamma(G)$ where $n=|G|$. Hence we may assume that $G$ is non-cyclic.

If $G$ has two distinct minimal normal subgroups, $A$ and $B$, then $G$ embeds in $G / A \times G / B$ and so is Abelian. Since $G$ is not cyclic, the Frattini subgroup of $G$ must be trivial. Thus, $G$ is a direct product of cyclic groups of prime order. It follows easily that $G$ is elementary Abelian of order $p^{2}$ for some prime $p$. Then each vertex in $\Gamma(G)$ has degree $p^{2}-p$ and so there is a Hamiltonian cycle in $\Gamma(G)$ by Pósa's criterion.

So we may assume that $G$ has a unique minimal normal subgroup $M$. It follows that $M$ is an elementary Abelian $p$-group for some prime $p$ and the cyclic group $G / M$ acts faithfully and irreducibly on $M$. Since the cyclic group $G / M$ acts faithfully and irreducibly on $M$, the integers $|G / M|$ and $|M|$ are coprime. By the SchurZassenhaus Theorem, $G$ is a split extension of $M$ by $H=G / M$ and all complements of $M$ in $G$ are conjugate. Hence $H$ can be considered to be an irreducible subgroup of a Singer cycle on $M$. It follows that $G$ is a primitive Frobenius group. Put $m=|M|$. Let $H_{1}, \ldots, H_{m}$ be the distinct conjugates of $H$ in $G$. Notice that $m \geq 3$. For each $i$ with $1 \leq i \leq m$ the cyclic group $H_{i}$ is maximal in $G$.

Put $n=|H|$ and let $h$ be a generator of $H_{m}$. For each $k$ with $1 \leq k \leq m$ let $v_{k}$ be the unique element of $M$ with $v_{k}{ }^{-1} H_{m} v_{k}=H_{k}$. Let $j$ be an arbitrary positive integer with $1 \leq j \leq m \cdot n$. If $j$ is a multiple of $n$, then set $g_{j}=v_{k}$ where $k$ is such that $k \equiv j(\bmod m)$. Otherwise, if $j$ is not a multiple of $n$, then set $g_{j}=v_{k}{ }^{-1} h^{i} v_{k}$ where $i$ and $k$ are so that $i \equiv j(\bmod n)$ and $k \equiv j(\bmod m)$. Then $g_{m \cdot n}$ is the 
identity element of $G$ and $g_{1}, \ldots, g_{m \cdot n-1}$ are precisely the non-identity elements of $G$. We claim that the vertices $g_{1}, \ldots, g_{m \cdot n-1}, g_{1}$ determine a Hamiltonian cycle in $\Gamma(G)$. To show this claim, let $x$ and $y$ be two consecutive elements in the previous list and set $L=\langle x, y\rangle$. By construction, $L$ projects onto $G / M$ via the natural homomorphism from $G$ to $G / M$ but $L$ is not conjugate to $H_{1}$. From this it follows that $L$ cannot be contained in a maximal subgroup containing $M$ (of the form $M \rtimes K$ for $K$ a maximal subgroup of $H$ ) and $L$ cannot lie in any complement of $M$ in $G$. Since $G$ is an affine primitive permutation group with $(|M|,|H|)=1$, it follows, from the Schur-Zassenhaus Theorem, that $L$ is contained in no maximal subgroup of $G$, hence $L=G$.

\section{Groups of Lie Type}

In this section it is shown that the graph $\Gamma(G)$ satisfies Pósa's criterion (and hence contains a Hamiltonian cycle) for every sufficiently large finite simple group $G$ of Lie type.

By a random element of a non-empty finite set $S$ we mean an element chosen uniformly from $S$. For a finite group $G$ let $P(G)$ be the probability that a random pair of elements of $G$ generate $G$. For a finite group $G$ and an element $x \in G$, define $P_{x}(G)$ to be the probability that $x$ and a randomly chosen element $y$ generate $G$. Note that for a non-identity element $x$ in a non-cyclic finite group $G$ the number $P_{x}(G)|G|$ is the degree of the vertex in $\Gamma(G)$ corresponding to $x$ in $G$. Let $m(G)$ denote the minimal index of a proper subgroup in a finite simple group $G$.

The following two theorems are needed.

Theorem 4.1 (Liebeck, Shalev, [13]). There exists a universal constant $c_{1}$ so that $1-\left(c_{1} / m(G)\right)<P(G)$ for an arbitrary finite simple group $G$.

Theorem 4.2 (Fulman, Guralnick, [6]). There exists a universal positive constant $c_{2}$ so that $c_{2}<P_{x}(G)$ for an arbitrary non-identity element $x$ in a finite simple group $G$ of Lie type.

Let $G$ be a finite simple group of Lie type. Let $m+1$ be the order of $G$ and let $d_{1} \leq \ldots \leq d_{m}$ be the list of vertex degrees of the graph $\Gamma(G)$. Let $t$ be the largest index (with $1 \leq t \leq m$ ) for which $d_{t}<(m+1) / 2$. (We may assume that such a $t$ exists for otherwise $\Gamma(G)$ satisfies Pósa's criterion and so there exists a Hamiltonian cycle in $\Gamma(G)$.) Then

$$
(m+1)^{2} P(G)=\sum_{i=1}^{m} d_{i}<t(m+1) / 2+(m-t)(m+1) .
$$

¿From this inequality and Theorem 4.1 we see that $t$ must satisfy

$$
t<\frac{2 c_{1}(m+1)}{m(G)}
$$

where $c_{1}$ is as in Theorem 4.1. Hence, if $G$ is sufficiently large, then we have

$$
t<c_{2}(m+1) \text {. }
$$

From this and Theorem 4.2 we find that $\Gamma(G)$ satisfies Pósa's criterion and hence contains a Hamiltonian cycle for $G$ sufficiently large.

\section{Alternating Groups}

In this section it is shown that for every sufficiently large alternating group $A_{n}$ the graph $\Gamma\left(A_{n}\right)$ satisfies Pósa's criterion (and hence contains a Hamiltonian 
cycle). This result together with the result of the previous section provides a proof for Theorem 1.2.

Let $G$ be a subgroup of $S_{n}$.

Theorem 5.1 (Babai, Hayes, [1]). For every $\epsilon>0$ there exists $\delta>0$ and a threshold $n_{0}$ such that for every $n \geq n_{0}$, if $G \leq S_{n}$ has fewer than $[\delta n]$ fixed points then the probability that $G$ and a random element $\sigma \in S_{n}$ generate $A_{n}$ or $S_{n}$ is at least $1-\epsilon$.

The following direct consequence of Theorem 5.1 is also indicated in [1]. Let $\pi$ be a permutation in $A_{n}$.

Corollary 5.2. For every $\epsilon>0$ there exists $\delta>0$ and a threshold $n_{0}$ such that for every $n \geq n_{0}$, if $\pi \in A_{n}$ has fewer than $[\delta n]$ fixed points then the probability that $\pi$ and a random element $\sigma \in A_{n}$ generate $A_{n}$ is at least $1-\epsilon$.

In this section, let $\delta$ and $n_{0}$ be positive numbers which fulfill the statement of Corollary 5.2 for $\epsilon=1 / 2$. Also, in this section, assume that $n \geq n_{0}$. Let $A(n)$ be the set of those even permutations of degree $n$ which fix fewer than $[\delta n]$ points and let $B(n)$ be $A_{n} \backslash A(n)$. Clearly, $|B(n)| \leq n ! /([\delta n]) !$.

Theorem 5.3. Let $n \geq 8$. The degree of every vertex in $\Gamma\left(A_{n}\right)$ is at least $n ! /\left(10 n^{3}\right)$.

Proof. This follows from the proof of Proposition 7.1 of [9].

By Corollary 5.2, our choice of $\epsilon$, and Theorem 5.3, the graph $\Gamma\left(A_{n}\right)$ satisfies Pósa's criterion provided that $n$ is at least $\max \left\{8, n_{0}\right\}$ and satisfies the inequality

$$
n ! /\left(10 n^{3}\right) \geq(n ! /([\delta n]) !)+1 \geq|B(n)|+1 .
$$

Hence $\Gamma\left(A_{n}\right)$ is indeed Hamiltonian for sufficiently large $n$.

\section{Symmetric Groups}

In this section Theorem 1.3 is proved.

Let $\Gamma(G)$ be defined as usual. If $G=S_{n}$, let $\Gamma_{b}(G)$ denote the bipartite subgraph of $\Gamma(G)$ obtained by throwing out edges between elements that are not in $H:=A_{n}$. Using a variation on the ideas in $[4, \S 6]$, we prove:

Theorem 6.1. Assume that $n>15$. Then the minimal degree of any vertex in $\Gamma_{b}(G)$ is at least $n ! / n^{3}$.

Proof. First suppose that $n=2 m$ is even. Let $C$ be the conjugacy class of products of two cycles of lengths $m+1$ and $m-1$ if $m$ is even and of lengths $m+2$ and $m-2$ if $m$ is odd. If $s \in G \backslash H$, then the probability that a random element of $C$ and $s$ generate $G$ is greater than $1 / 2\left[4\right.$, Lemma 6.4]. Since $|C| \geq(n !) / m^{2}$, it follows that the vertex degree of $s$ is at least $n ! / n^{2}$.

Let $C$ be a conjugacy class (of $G$ ) consisting of three cycles of lengths $d_{1}<d_{2}<$ $d_{3}$ with $d_{1}=[n / 3]-1$. More precisely, if $n=3 m$ then let $d_{1}=m-1, d_{2}=m$, $d_{3}=m+1$; if $n=3 m+1$ then let $d_{1}=m-1, d_{2}=m, d_{3}=m+2$; and if $n=3 m+2$ then let $d_{1}=m-1, d_{2}=m+1, d_{3}=m+2$. Note that no element of $C$ lies inside an imprimitive transitive subgroup. Note also that the elements of $C$ have the property that some specific power of any given element of $C$ moves exactly $d_{2}$ points and in fact is a cycle of precisely that size. By a result of Williamson [21], it follows that no element of $C$ lies inside a primitive subgroup of $G$. Hence we conclude that the only maximal subgroups of $G$ containing an element of $C$ are the obvious intransitive subgroups. 
Let $1 \neq h \in H$. We want to show that the number of edges in $\Gamma_{b}(G)$ connecting $h$ and an element of $C$ is at least $n ! / n^{3}$ whenever $n>15$. Clearly, we can replace $h$ by a power of $h$ and assume that $h$ has prime order. If $h=h_{1} h_{2}$ is a product of two disjoint permutations both in $H$ then the number of edges from $h_{1}$ to an element of $C$ is at most the number of edges from $h$ to an element of $C$. (This is because if $x \in C$ then $\left\langle h_{1}, x\right\rangle$ is transitive implies that $\langle h, x\rangle$ is transitive.) So we may assume that $h$ is either a $p$-cycle with $p$ an odd prime or a product of two disjoint transpositions. The probability that a random element of $C$ and such an $h$ is intransitive is roughly at most $3(2 / 3)^{3}$ and is always less than 0.9 . Thus, the probability that $h$ and a random element of $C$ generate $G$ is at least 0.1 . Thus, the degree of the vertex $h$ is at least $|C| / 10 \geq n ! / n^{3}$ (note that for $x \in C$, we have $\left.\left|C_{G}(x)\right|<(n / 3)^{3}\right)$.

Now suppose that $n$ is odd. Let $C$ be the conjugacy class of $n$-cycles. If $s \in G \backslash H$ is not a transposition, then the probability that a random element of $C$ and $s$ generate $G$ is greater than $2 / 3$ [4, Proposition 6.8]. Thus, the vertex degree of $s$ is at least $2|C| / 3=2(n !) / 3 n$. Suppose that $s$ is a transposition. If $x \in C$, then $\langle x, s\rangle=G$ unless $\langle x, s\rangle$ is imprimitive.

We reverse the computation. Fix $x \in C$. Take it to be $(1,2, \ldots, n)$. Note that $x$ fixes a unique partition with block size $d$ for each divisor of $n$. Let $s=(1 j)$. Then $\langle x, s\rangle=G$ if and only if $\operatorname{gcd}(n, j-1)=1$. So the probability that a random transposition and $x$ generate $G$ is at least $1 / n$, whence the probability that $s$ and a random element of $C$ generate $G$ is at least $1 / n$. Thus, the degree of the vertex $s$ is at least $|C| / n=(n !) / n^{2}$.

Now suppose that $1 \neq h \in H$. Let $C$ be the conjugacy class of elements that are a product of an $m$-cycle and an $m+1$-cycle where $n=2 m+1$. Then the probability that a random element of $C$ and $s$ generate $G$ is greater than $1 / 2$ [4, Lemma 6.5]. Thus, the degree of the vertex $s$ is at least $(n !) /\left(2 n^{2}\right)$.

Two direct consequences of Theorem 5.1 are

Corollary 6.2. For every $\epsilon_{1}>0$ there exists $\delta_{1}>0$ and a threshold $n_{1}$ such that for every $n \geq n_{1}$, if $\pi \in S_{n} \backslash A_{n}$ has fewer than $\left[\delta_{1} n\right]$ fixed points then the probability that $\pi$ and a random element $\sigma \in S_{n}$ generate $S_{n}$ is at least $1-\epsilon_{1}$.

Corollary 6.3. For every $\epsilon_{2}>0$ there exists $\delta_{2}>0$ and a threshold $n_{2}$ such that for every $n \geq n_{2}$, if $\pi \in A_{n}$ has fewer than $\left[\delta_{2} n\right]$ fixed points then the probability that $\pi$ and a random element $\sigma \in S_{n}$ generate $S_{n}$ is at least $(1 / 2)-\epsilon_{2}$.

Let $\delta_{1}, n_{1}$ and $\delta_{2}, n_{2}$ be positive numbers satisfying the statements of Corollaries 6.2 and 6.3 for $\epsilon_{1}=1 / 5$ and $\epsilon_{2}=1 / 5$ respectively. Let $\delta$ be the minimum of $\delta_{1}$ and $\delta_{2}$ and let $m_{0}$ be the maximum of $n_{1}$ and $n_{2}$. Unless otherwise stated assume that $n \geq m_{0}$. Let $A_{1}(n)$ and $A_{2}(n)$ be the set of elements of $S_{n} \backslash A_{n}$ and $A_{n}$ respectively fixing less than $[\delta n]$ points. Let $B_{1}(n)$ and $B_{2}(n)$ be $\left(S_{n} \backslash A_{n}\right) \backslash A_{1}(n)$ and $A_{n} \backslash\left(A_{2}(n) \cup\{1\}\right)$ respectively. Clearly,

$$
\left|B_{i}(n)\right| \leq \frac{n !}{2([\delta n]) !}
$$

for $i=1,2$.

Lemma 6.4. For sufficiently large $n$, the set $S_{n} \backslash A_{n}$ spans a complete subgraph in the graph $\mathrm{cl}^{(3)}\left(\Gamma\left(S_{n}\right)\right)$. Moreover, for $n$ sufficiently large, every vertex in $A_{1}(n)$ is connected to every other vertex and every vertex in $B_{1}(n)$ is connected to at least $(n ! / 2)-1+\left(n ! / n^{3}\right)$ other vertices in the graph $\mathrm{cl}^{(3)}\left(\Gamma\left(S_{n}\right)\right)$. 
Proof. Let $n \geq \max \left\{m_{0}, 15\right\}$. Set $\Gamma_{0}=\Gamma\left(S_{n}\right)$. We claim that in the graph $\Gamma_{1}=$ $\operatorname{cl}\left(\Gamma\left(S_{n}\right)\right)$ the set $A_{1}(n)$ spans a complete subgraph and every vertex in $A_{1}(n)$ is connected to every vertex in $A_{2}(n)$.

For the first claim notice that for any $u, v$ in $A_{1}(n)$ we have

$$
d\left(\Gamma_{0}, u\right)+d\left(\Gamma_{0}, v\right)>(8 / 5)(n !-1)>n !-1 .
$$

For the latter claim let $u \in A_{1}(n)$ and $v \in A_{2}(n)$. Then

$$
d\left(\Gamma_{0}, u\right)+d\left(\Gamma_{0}, v\right)>(11 / 10)(n !-1)>n !-1 .
$$

Now we claim that, for sufficiently large $n$, in the graph $\Gamma_{2}=\mathrm{cl}^{(2)}\left(\Gamma\left(S_{n}\right)\right)$ every vertex in $A_{1}(n)$ is connected to every other vertex in the graph. Let $u \in A_{1}(n)$ and let $v \in B_{1}(n) \cup B_{2}(n)$ be arbitrary. Then, by Theorem 6.1 and by the observation made before the statement of the lemma,

$$
d\left(\Gamma_{1}, u\right)+d\left(\Gamma_{1}, v\right)>n !-2-\left|B_{1}(n) \cup B_{2}(n)\right|+n ! / n^{3}>n !-1 .
$$

Next we claim that, in the graph $\Gamma_{3}=\mathrm{cl}^{(3)}\left(\Gamma\left(S_{n}\right)\right)$, every vertex in $B_{1}(n)$ is connected to every other vertex in $B_{1}(n)$. Let $u$ and $v$ be two arbitrary elements from $B_{1}(n)$. Then, again by Theorem 6.1 and by the observation made before the statement of the lemma,

$$
d\left(\Gamma_{2}, u\right)+d\left(\Gamma_{2}, v\right) \geq 2\left|A_{1}(n)\right|+(2 n !) /\left(n^{3}\right)>n !-1 .
$$

Finally, it follows from the above and from Theorem 6.1 that every vertex in $B_{1}(n)$ is connected to at least $(n ! / 2)-1+\left(n ! / n^{3}\right)$ other vertices in the graph $\Gamma_{3}$.

By Theorem 2.1, the following lemma finishes the proof of Theorem 1.3.

Lemma 6.5. For sufficiently large $n$ the graph $\mathrm{cl}^{(3)}\left(\Gamma\left(S_{n}\right)\right)$ satisfies Chvátal's criterion. In particular, the graph $\mathrm{cl}^{(3)}\left(\Gamma\left(S_{n}\right)\right)$ contains a Hamiltonian cycle.

Proof. Put $\Gamma_{3}=\mathrm{cl}^{(3)}\left(\Gamma\left(S_{n}\right)\right)$. Let $d_{1} \leq \ldots \leq d_{n !-1}$ be the list of vertex degrees of the graph $\Gamma_{3}$. Let $k$ be a positive integer at most $n ! / 2$. It is sufficient to show that $d_{n !-1-k} \geq n !-1-k$. Since every vertex in $A_{1}(n)$ has maximum possible degree in $\Gamma_{3}$ by Lemma 6.4 , the claim is clear for positive integers $k$ satisfying

We may now assume that

$$
k \leq \frac{n !}{2}-\frac{n !}{2([\delta n]) !} .
$$

$$
\frac{n !}{2}-\frac{n !}{2([\delta n]) !}<k<\frac{n !}{2} .
$$

But then by Theorem 6.1 and Lemma 6.4 , we have

$$
d_{n !-1-k} \geq \frac{n !}{2}-1+\frac{n !}{n^{3}}>n !-1-\frac{n !}{2}+\frac{n !}{2([\delta n]) !} \geq n !-1-k .
$$

\section{Wreath Products}

Let $S$ be a non-abelian finite simple group and let $C_{m}$ be the cyclic subgroup of $S_{m}$ generated by the cyclic permutation $\sigma=(1,2, \ldots, m)$, with $m=p^{t}$ a prime power. Consider the wreath product $G=S$ < $C_{m}$. Denote the base subgroup of $G$ by $N=S_{1} \times \cdots \times S_{m}$ and let $\pi_{i}: N \rightarrow S_{i}$ be the projection on the $i$-th factor. Moreover let $A=\operatorname{Aut}(S), r=p^{t-1}, u=r+1$ and $\Lambda$ the set $\{1+r i \mid 0 \leq i \leq p-1\}$. 
Lemma 7.1. A subgroup $H$ of $G$ coincides with $G$ if the following properties are satisfied:

(1) $H N / N \cong C_{m}$;

(2) $\pi_{i}(H \cap N) \cong S$ for some $i$;

(3) there exists $\left(y_{1}, \ldots, y_{m}\right) \in H \cap N$ and $a, b \in \Lambda$ such that $y_{a}$ and $y_{b}$ are not A-conjugate.

Proof. If $H$ satisfies the first two conditions, then $H \cap N$ is a subdirect product of $N=S_{1} \times \cdots \times S_{m}$. If $H \neq G$ then $H \cap N \leq \prod_{j} D_{j}$ where $D_{j}=\left\{\left(s, s^{b_{2}}, \ldots, s^{b_{v}}\right) \in\right.$ $\left.\prod_{i \in B_{j}} S_{i} \mid s \in S, b_{i} \in A\right\}$ is a diagonal subgroup of $\prod_{i \in B_{j}} S_{i}$ and the subsets $B_{j}$ form a system of blocks for the action of $C_{m}$ on $\{1, \ldots, m\}$, with $\left|B_{j}\right| \neq 1$. To conclude note that for any choice of $B_{j}$ 's, $a$ and $b$ belong to the same block.

Lemma 7.2. Let $i$ be an integer not divisible by $p, \rho=\sigma^{i}, \tau=\sigma^{r}$, and $g=$ $\left(x_{1}, \ldots, x_{m}\right) \tau \in G$ where $\left(x_{1}, \ldots, x_{m}\right) \in N$. The probability that there is an edge in $\Gamma(G)$ between $g$ and a randomly chosen element in the coset $\rho N$ is at least $\eta$, where $\eta$ is the probability that two randomly chosen elements from $S$ generate $S$ and are not A-conjugate.

Proof. It is not restrictive to assume that $x_{i}=1$ for each $i>r$ (just substitute $g$ with a conjugate $g^{x}$ for a suitable choice of $\left.x \in N\right)$. Now consider $h=\rho\left(y_{1}, \ldots, y_{m}\right)$. There exists $k<m$ and $\left(h_{1}, \ldots, h_{m}\right) \in N$ such that

$$
h^{k}=\left(\rho\left(y_{1}, \ldots, y_{m}\right)\right)^{k}=\tau^{-1}\left(h_{1}, \ldots, h_{m}\right) .
$$

Let $H$ be the subgroup generated by $g$ and $h$ (which clearly satisfies the first condition of Lemma 7.1). Notice that $H \cap N$ contains $w=\left(x_{1} h_{1}, \ldots, x_{r} h_{r}, h_{u}, \ldots, h_{m}\right)$ and $w^{g}$. Notice also that $\pi_{u}\left(w^{g}\right)=h_{1} x_{1}$. In particular the second and third condition of Lemma 7.1) are satisfied if $h_{u}, h_{1} x_{1}$ are not $A$-conjugate and generate $S$. Hence there are $\eta|S|^{2}$ possible choices for $\left(h_{1}, h_{u}\right)$. Now notices that there exists two distinct subsets $X_{1}$ and $X_{u}$ of $\{1, \ldots, m\}$, of cardinality $k$ such that, for $i \in\{1, u\}, h_{i}$ is the product of the $k$ elements $y_{j}$ with $j \in X_{i}$ (in a suitable order); take $a \in X_{1} \backslash X_{u}$ and $b \in X_{u} \backslash X_{1}$ : to obtain a prescribed value for $h_{1}, h_{u}$, we can choose $y_{i}$ as we like for $i \notin\{a, b\}$, then choose $y_{a}$ and $y_{b}$ in order to get the wanted values. So we find $\eta|N|$ suitable choices for the elements $y_{i}$.

Corollary 7.3. If $g \in G \backslash N$, then the degree of $g$ as a vertex of $\Gamma(G)$ is at least $\phi(m)|N| \eta=p^{t-1}(p-1)|N| \eta$.

With similar arguments it can be proved that we have at least $\eta|G|$ edges from the elements that generate $G$ modulo $N$.

Lemma 7.4. Let $g=\left(x_{1}, \ldots, x_{m}\right) \sigma \in G$. The probability that there is an edge in $\Gamma(G)$ between $g$ and a randomly chosen element of $G$ is at least $\eta$.

Proof. It is not restrictive (by substituting $g$ with a suitable conjugate) to assume that $x_{1}=\cdots=x_{m-1}=1$. Take an arbitrary element $x=\left(y_{1}, \ldots, y_{m}\right) \sigma^{i} \in G$; there exist $k \in \mathbb{N}$ and $\left(z_{1}, \ldots, z_{m}\right) \in N$ with $g^{k}=\sigma^{-i}\left(z_{1}, \ldots, z_{m}\right)$. Clearly $\langle g, x\rangle=G$ if and only if $\left\langle g,\left(y_{1} z_{1}, \ldots, y_{m} z_{m}\right)\right\rangle=G$ : in particular $\langle g, x\rangle=G$ if we choose $y_{1}, y_{u}$ so that $y_{1} z_{1}$ and $y_{u} z_{u}$ are not $A$-conjugate and generate $S$.

We need now some information on the behavior of $P_{n}(G)$ when $n \in N$.

Lemma 7.5. Assume that $n=\left(x_{1}, \ldots, x_{m}\right) \in N$, with $n \neq 1$. Choose $i \in\{1, \ldots, m\}$ with the property that $P_{x_{i}}(S) \geq P_{x_{j}}(S)$ for each $1 \leq j \leq m$ and let $x=x_{i}$. Given 
a generator $\tau$ of $C_{m}=\langle\sigma\rangle$, the number of edges connecting $n$ with elements of the coset $N \tau$ is at least $|N| \mu$ with

$$
\mu=\max \left(P_{x}(S)-\frac{\left|C_{A}(x)\right|}{|S|}, \frac{P_{x}(S)\left|C_{S}(x)\right|}{|S|} \rho_{x}\right)
$$

with $\rho_{x}=1$ if $C_{A}(x) S=A$ and $\rho_{x}=0$ otherwise.

Proof. It is not restrictive to assume that $i=1$ and $\tau=\sigma$. First we claim that the number of edges connecting $n$ with elements of the coset $N \sigma$ is at least $|N| \mu_{1}$ with

$$
\mu_{1}=P_{x}(S)-\frac{\left|C_{A}(x)\right|}{|S|} .
$$

It suffices to prove that, for any $y_{2}, \ldots, y_{m} \in S^{m-1}$, there exist at least $\mu_{1}|S|$ choices for $y_{1}$ such that if $g=\left(y_{1}, \ldots, y_{m}\right) \sigma$ then $\langle n, g\rangle=G$. We have $g^{m}=\left(h_{1}, \ldots, h_{m}\right)$ with $h_{1}=y_{1} y_{2} \cdots y_{m}$. In particular the second condition of Lemma 7.1 is satisfied if $x$ and $h_{1}$ generate $S$ and there are at least $|S| P_{x}(S)$ choices for $y_{1}$ for which this is ensured. If there exist $\lambda \in \Lambda$ with $x_{1}$ and $x_{\lambda}$ not $A$-conjugate, then the third condition is automatically satisfied and we are done. Otherwise for each $1 \leq i \leq p-1$ there exist $\alpha_{i} \in A$ with $x_{i r+1}=x^{\alpha_{i}}$. In this case to be sure that $H=\langle g, n\rangle=G$ we need an extra condition on $y_{1}$ to avoid that $\pi_{\Lambda}(H \cap N)=\left(s, s^{\beta_{1}}, \ldots, s^{\beta_{p-1}}\right)$ with $\beta_{i} \in A$. Assume that this is the case. Since $\left(x, x_{r+1}, \ldots, x_{r(p-1)+1}\right)=\pi_{\Lambda}(n)$, we must have $\beta_{i} \in C_{A}(x) \alpha_{i}$. Let $g^{r}=\left(k_{1}, \ldots, k_{m}\right) \sigma^{r}$ and let $\epsilon$ be the $p$-cycle $(1, r+$ $1, \ldots, r(p-1))$. Since $g^{r}$ normalizes $H \cap N$, we have that $\left(k_{1}, k_{r+1}, \ldots, k_{r(p-1)+1}\right) \epsilon$ normalizes $\pi_{\Lambda}(H \cap N)=\left(s, s^{\beta_{1}}, \ldots, s^{\beta_{p-1}}\right)$. In particular, setting $z=\beta_{p-1} k_{r(p-1)+1}$, we have $z \beta_{1}=k_{1}$ and $z \beta_{i}=\beta_{i-1} k_{(i-1) r+1}$ for each $2 \leq i \leq p-1$ and consequently

$$
z^{p}=k_{1} k_{r+1} \cdots k_{r(p-1)+1}=h_{1} .
$$

Since $k_{r(p-1)+1}$ depends only on $y_{2}, \ldots, y_{n}$, the set $\Delta=\left\{\left(t \alpha_{p-1} k_{r(p-1)+1}\right)^{p} \mid t \in\right.$ $\left.C_{A}(x)\right\}$ is independent from $y_{1}$. If we choice $y_{1}$ such that $\left\langle x, h_{1}\right\rangle=S$ and $h_{1} \notin \Delta$, then $\langle g, n\rangle=G$. Clearly the number of $y_{1}$ for which $h_{1}$ satisfies the two previous conditions is at most

$$
|S|\left(P_{x}(S)-\frac{\left|C_{A}(x)\right|}{|S|}\right) .
$$

This concludes the proof of the first claim. Now we want to show that the number of edges connecting $n$ with elements of the coset $N \sigma$ is at least $|N| \mu_{2}$ with

$$
\mu_{2}=\frac{P_{x}(S)\left|C_{S}(x)\right|}{|S|} \rho_{x} .
$$

Note that there are at least $\mu_{2}|N|$ choices of $\left(y_{1}, \ldots, y_{m}\right)$ so that $\left\langle h_{1}, x\right\rangle=S$ and $k_{r(p-1)+1} \in \alpha_{p-1}^{-1} C_{A}(x)$. We claim that for any of these choices, $g=\left(y_{1}, \ldots, y_{m}\right) \sigma$ generates $G$ together with $n$. By the argument that we have used above, and under the same notations, it suffices to prove that $z^{p} \neq h_{1}$. Notice that $z=$ $\beta_{p-1} k_{r(p-1)+1} \in \beta_{p-1} \alpha_{p-1}^{-1} C_{A}(x) \leq C_{A}(x)$, hence $z^{p}=h_{1}$ would imply $\left[h_{1}, x\right]=1$, against $\left\langle h_{1}, x\right\rangle=S$.

Lemma 7.6. Let $S$ be a non-abelian finite simple group and let $c(S)$ be the maximal size of a conjugacy class of $S$. Then $\lim _{|S| \rightarrow \infty}(c(S)|\operatorname{Out}(S)|) /|S|=0$.

Proof. If $S$ is a finite simple group of Lie type, then this follows by [7, Theorem 1.4]. If $S=A_{n}$ for $n>6$, then $(c(S)|\operatorname{Out}(S)|) /|S| \leq 4 / n$.

Lemma 7.7. Let $\eta$ be as above. Then $\lim _{|S| \rightarrow \infty} \eta=1$. 
Proof. Let $S$ be a non-abelian finite simple group and let $A$ be the automorphism group of $S$. Notice that $\eta \geq 1-p-q$ where $p$ is the probability that a random pair of elements of $S$ does not generate $S$ and $q$ is the probability that a random pair of elements of $S$ is $A$-conjugate. By Theorem 4.1), $p$ tends to 0 as $|S|$ tends to infinity. Thus, to prove the lemma, it is sufficient to show that $q$ tends to 0 as $|S|$ tends to infinity.

Let $k$ be the number of $A$-conjugacy classes of elements of $S$ and let $a_{1}, \ldots, a_{k}$ be the corresponding orbit sizes with $a_{1} \geq \ldots \geq a_{k}$. We have $q=\left(\sum_{i=1}^{k} a_{i}{ }^{2}\right) /|S|^{2}$.

Put $a=a_{1}, n=|S|$, and $b=n-[n / a] a$. We claim that $q \leq\left([n / a] a^{2}+b^{2}\right) / n^{2}$. Before verifying this claim, let us show how our lemma would follow.

Indeed,

$$
q \leq \frac{[n / a] a^{2}+b^{2}}{n^{2}}<\frac{a}{n}\left(1+\frac{a}{n}\right) \leq \frac{c(S)|\operatorname{Out}(S)|}{n}\left(1+\frac{c(S)|\mathrm{Out}(S)|}{n}\right)
$$

and, by Lemma 7.6, the right-hand-side of this inequality tends to 0 as $n$ tends to infinity, hence $q$ must tend to 0 .

Finally, for the proof of our claim, observe that if $x$ and $y$ are two positive integers with $x \leq y$, then $(x-1)^{2}+(y+1)^{2}=x^{2}+y^{2}+2+2(y-x)>x^{2}+y^{2}$. This means that, starting from the list $a=a_{1}, \ldots, a_{k}$, we may derive a sequence of lists by replacing two elements $x$ and $y$ of the previous list by $x-1$ and $y+1$ in the next list, whenever $1 \leq x \leq y<a$. This way, the last list of non-negative integers will be $a, \ldots, a, b, 0, \ldots, 0$ where $b=n-[n / a] a$.

We are now in the position to prove Theorem 1.4.

We divide the vertices of $\Gamma(G)$ into three disjoint subsets:

- $V_{1}$ is the set of vertices corresponding to elements $\left(y_{1}, \ldots, y_{m}\right) \tau$ with $|\tau|=$ $m$;

- $V_{2}$ is the set of vertices corresponding to elements $\left(y_{1}, \ldots, y_{m}\right) \tau$ with $1<$ $|\tau|<m$

- $V_{3}$ is the set of vertices corresponding to the non trivial elements of the base group $N$ of the wreath product.

Let $\Gamma_{0}=\Gamma(G)$ and $\Gamma_{i}=\mathrm{cl}^{(i)}(\Gamma(G))$ for $i \geq 1$. By Lemma 7.4 and Corollary 7.3, if $u \in V_{1}$ and $v \in V_{1} \cup V_{2}$, then

$$
d\left(\Gamma_{0}, u\right)+d\left(\Gamma_{0}, v\right) \geq \eta|G|+\eta|G|\left(1-\frac{1}{p}\right) \geq 3 \eta|G| / 2 .
$$

Since $\eta$ tends to 1 as $|S|$ tends to infinity (by Lemma 7.7), we deduce that if $|S|$ is large enough then any vertex in $V_{1}$ is connected to any other vertex in $V_{1} \cup V_{2}$ in the first closure $\Gamma_{1}$. But then, if $v_{1}, v_{2} \in V_{2}$, then

$$
d\left(\Gamma_{1}, v_{1}\right)+d\left(\Gamma_{1}, v_{2}\right) \geq 2\left|V_{1}\right|=2\left(1-\frac{1}{p}\right)|G| \geq|G|
$$

which means that $\Gamma_{2}$ induces a complete subgraph on $V_{1} \cup V_{2}$.

To complete the proof we need different arguments for the Lie and alternating cases.

First assume that $S$ is a group of Lie type. By Theorem 4.2 and the fact that $\max _{x \in S, x \neq 1}\left|C_{A}(x)\right| /|S|$ tends to 0 as $|S|$ tends to infinity, we deduce that there exists a positive constant $c_{3}$ such that, if $S$ is large enough then, for any $x \in S \backslash\{1\}$,

$$
P_{x}(S)-\frac{\left|C_{A}(x)\right|}{|S|} \geq c_{3} .
$$


By Lemmas 7.4 and 7.5, for any $u \in V_{1}$ and $u \in V_{3}$ we have

$$
d\left(\Gamma_{0}, u\right)+d\left(\Gamma_{0}, v\right) \geq \eta|G|+c_{3}|G|\left(1-\frac{1}{p}\right)=\left(\eta+c_{3}\left(1-\frac{1}{p}\right)\right)|G| .
$$

If $|S|$ is large enough, then $\eta+c_{3}(1-1 / p) \geq 1$ : in this case any vertex in $V_{1}$ is connected to any other vertex in $V_{3}$ in the first closure $\Gamma_{1}$, and this implies that $\Gamma_{2}$ is a complete graph.

We remain with the alternating groups. In this case we need to use the BabaiHayes Theorem. Let $\delta$ and $n_{0}$ be positive numbers which fulfill the statement of Corollary 5.2 for $\epsilon=1 / 2$. Let $A(n)$ be the set of those even permutations of degree $n$ which fix fewer than $[\delta n]$ points. We divide $V_{3}$ into two disjoint subsets: $W_{1}$ is the set of elements $\left(y_{1}, \ldots, y_{m}\right)$ of $N$ with the property that $y_{i} \in A(n)$ for some $1 \leq i \leq m ; W_{2}=V_{3} \backslash W_{1}$. Since $P_{x}(S) \geq 1 / 2$ for each $x \in A(n)$, arguing as in the case of groups of Lie type we deduce that $\Gamma_{2}$ induces a complete subgraph on $V_{1} \cup V_{2} \cup W_{1}$. Let now $w=\left(y_{1}, \ldots, y_{m}\right) \in W_{2}$ and assume that $y=y_{i}$ has the property that $P_{y_{i}}(S) \geq P_{y_{j}}(S)$ for each $1 \leq j \leq m$. Let now $p=P_{y}(S)$ and $c=\left|C_{A}(y)\right| /|S|$ and consider

$$
\mu=\max \left(P_{y}(S)-\frac{\left|C_{A}(y)\right|}{|S|}, \frac{P_{y}(S)\left|C_{S}(y)\right|}{|S|}\right) .
$$

If $c \leq p / 2$ then $\mu \geq p / 2 \geq p^{2} / 4$, otherwise, if $c \geq p / 2$, we again have $\mu \geq p^{2} / 4$. Moreover, by Theorem 5.3, $p \geq 1 /\left(5 n^{3}\right)$, hence, by Lemma 7.5 ,

$$
d\left(\Gamma_{2}, w\right) \geq d\left(\Gamma_{0}, w\right) \geq \frac{\left(p^{t}-p^{t-1}\right)|N|}{100 n^{6}} .
$$

On the other hand

$$
\left|W_{2}\right| \leq|S-A(n)|^{m} \leq\left(\frac{n !}{[\delta n] !}\right)^{m} \leq|N|\left(\frac{2}{[\delta n] !}\right)^{m}
$$

so if $n$ is large enough, $d\left(\Gamma_{2}, w\right)>\left|W_{2}\right|$ for each $w \in W_{2}$. This means that $\Gamma_{2}$ satisfies Pósa's criterion, and hence contains a Hamiltonian cycle.

\section{Computer Calculations}

The main results of this paper hold for sufficiently large groups. In this section, we consider small groups and sporadic simple groups. In particular, we get a computational proof of Theorem 1.5. (Currently, we do not know how large the gap between small and sufficiently large is.)

Using the same computational methods as in [4, Section 2.5], we showed that the generating graphs of the following groups contain Hamiltonian cycles.

- Non-abelian simple groups of orders at most $10^{7}$,

- groups $G$ containing a unique minimal normal subgroup $N$ such that $N$ has order at most $10^{6}, N$ is nonsolvable, and $G / N$ is cyclic,

- alternating and symmetric groups on $n$ points, with $5 \leq n \leq 13$,

- sporadic simple groups and automorphism groups of sporadic simple groups.

More specifically, the generating graphs of the simple groups in this list satisfy Pósa's criterion, and for each non-simple group in this list a suitable iterated closure of the generating graph satisfies Pósa's criterion.

For that, we define the partial vertex degree of the non-identity element $s$ w. r. t. the conjugacy class $C$ as $d(\Gamma(G), s, C)=|\{x \in C ;\langle s, x\rangle=G\}|$. The vertex degree $d(\Gamma(G), s)$ equals $\sum_{C} d(\Gamma(G), s, C)$, where $C$ runs over the conjugacy classes of $G$, 
and a lower bound for $d\left(\Gamma(G), s, g^{G}\right)$ is given by $\left|g^{G}\right|-\sum_{M \in \mathcal{M}(G, s)}\left|g^{G} \cap M\right|$, where $\mathcal{M}(G, s)$ denotes the set of those maximal subgroups of $G$ that contain $s$.

The point is that these lower bounds can be computed easily if the primitive permutation characters of $G$ are known. This is the case when the table of marks of $G$ is available or if the character tables of $G$ and of all its maximal subgroups (and the necessary class fusions) are available, for example if $G$ is a sporadic simple group not equal to the Monster.

Defining partial vertex degrees for the iterated closures of $\Gamma(G)$ in the obvious way, we get $d\left(\operatorname{cl}(\Gamma(G)), s, g^{G}\right)=\left|g^{G}\right|$ if $d(\Gamma(G), s)+d(\Gamma(G), g) \geq|G|-1$, and $d\left(\operatorname{cl}(\Gamma(G)), s, g^{G}\right)=d\left(\Gamma(G), s, g^{G}\right)$ otherwise.

Note that lower bounds for the partial vertex degrees for the closures of $\Gamma(G)$ can be computed this way from lower bounds for the partial vertex degrees for $\Gamma(G)$.

If the primitive permutation characters of $G$ are not known then computing the (partial) vertex degrees directly, without character-theoretic computations, is usually faster than computing first the character information.

It turned out that this approach was sufficient to prove that Pósa's criterion holds for appropriate closures $\mathrm{cl}^{(i)}(\Gamma(G))$, for all groups $G$ listed above. See [3] for more information.

\section{Proof of Proposition 1.7}

Let us use the notations and assumptions of Proposition 1.7. Notice that if $G$ is generated by elements $x$ and $y$ then $d(x)+d(y) \leq \operatorname{dim}(V)$. Indeed, if $d(x)+d(y)>$ $\operatorname{dim}(V)$, then any non-trivial subspace of $U \cap W$ is $G$-invariant contradicting the irreducibility of $V$ where $U$ and $W$ are eigenspaces of $x$ and $y$ on $V$ of dimensions $d(x)$ and $d(y)$, respectively.

Let $n+1$ be the order of $G$ and let $x_{1}, \ldots, x_{n}, x_{n+1}=x_{1}$ be a Hamiltonian cycle in the graph $\Gamma(G)$. Then $d\left(x_{i}\right)+d\left(x_{i+1}\right) \leq \operatorname{dim}(V)$ for all $i$ with $1 \leq i \leq n$, hence

$$
\sum_{i=1}^{n} d\left(x_{i}\right)=\frac{1}{2} \sum_{i=1}^{n}\left(d\left(x_{i}\right)+d\left(x_{i+1}\right)\right) \leq \frac{n}{2} \operatorname{dim}(V)
$$

which is exactly what we wanted.

Acknowledgment. We thank L. Pyber for drawing our attention to [1].

\section{REFERENCES}

[1] Babai, L.; Hayes, T. P. The probability of generating the symmetric group when one of the generators is random. Publ. Math. Debrecen 69/3 (2006), 271-280.

[2] Bondy, J. A.; Chvatal, V. A method in graph theory. Discrete Math. 15 (1976), 111-136.

[3] Breuer, T. GAP computations concerning Hamiltonian cycles in the generating graphs of finite groups. arXiv:0911.5589.

[4] Breuer, T.; Guralnick, R. M.; Kantor, W. M. Probabilistic generation of finite simple groups, II. J. Algebra Vol. 320. 2, (2008), 443-494.

[5] Burness, T.; Guest, S.; Guralnick, R. M. Finite groups with positive spread, in preparation.

[6] Fulman, J.; Guralnick, R. M. The probability of generating an irreducible subgroup, preprint.

[7] Fulman, J.; Guralnick, R. M. Bounds on the number and sizes of conjugacy classes in finite Chevalley groups with applications to derangements. arXiv:0902.2238.

[8] The GAP Group, GAP - Groups, Algorithms, and Programming, Version 4.4; 2005, (http://www.gap-system.org).

[9] Guralnick, R. M.; Kantor, W. M. Probabilistic generation of finite simple groups. J. Algebra Vol. 234. 2, (2000), 743-792. 
[10] Guralnick, R. M.; Shalev, A. On the spread of finite simple groups. Combinatorica 23 (1) (2003), 73-87.

[11] Isaacs, I. M.; Keller, T. M.; Meierfrankenfeld, U.; Moretó, A. Fixed point spaces, primitive character degrees and conjugacy class sizes. Proc. Am. Math. Soc. 134 11, (2006), 3123-3130.

[12] The Kourovka Notebook. Unsolved problems in group theory. Sixteenth augmented edition, 2006. Edited by V. D. Mazurov and E. I. Khukhro.

[13] Liebeck, M. W.; Shalev, A. Simple groups, probabilistic methods, and a conjecture of Kantor and Lubotzky. J. Algebra 184 (1996), no. 1, 31-57.

[14] Lovász, L. Combinatorial problems and exercises. North-Holland, Amsterdam, 1979.

[15] Lucchini, A.; Maróti, A. Some results and questions related to the generating graph of a finite group. To appear in Proceedings of the Ischia Group Theory Conference 2008.

[16] Lucchini, A.; Maróti, A. On the clique number of the generating graph of a finite group. Proc. Am. Math. Soc. 137, No. 10, (2009), 3207-3217.

[17] Lucchini, A.; Maróti, A. On finite simple groups and Kneser graphs. J. Algebr. Comb. 30 No. 4, (2009), 549-566.

[18] Luczak, T.; Pyber, L. On random generation of the symmetric group. Combinatorics, Probability and Computing 2 (1993), 505-512.

[19] Neumann, P. M.; Vaughan-Lee, M. R. An essay on BFC groups. Proc. London Math. Soc. (3) 35 (1977), 213-237.

[20] Segal, D.; Shalev, A. On groups with bounded conjugacy classes. Quart. J. Math. Oxford 50 (1999), 505-516.

[21] Williamson, A. On primitive permutation groups containing a cycle. Math. Z. 130 (1973), 159-162.

Thomas Breuer, Lehrstuhl D für Mathematik, RWTH Aachen University, 52065 Aachen, Germany. E-mail address: sam@math.rwth-aachen.de

Robert M. Guralnick, Department of Mathematics, University of Southern California,Los Angeles, CA 90089-2532,USA. E-mail address: guralnic@usc.edu Andrea Lucchini, Dipartimento di Matematica Pura ed Applicata, Via Trieste 63,35121Padova, Italy. E-mail address: lucchini@math.unipd.it

Attila Maróti, MTA Alfréd Rényi Institute of Mathematics, Budapest, Hungary. E-mail address: maroti@renyi.hu

Gábor Péter Nagy, SZTE Bolyai Institute, Aradi Vértanúk tere 1, Szeged, 6720,

Hungary. E-mail address: nagyg@math.u-szeged.hu 